\title{
Photochemical transformations of 1,10-decylbischromones: effect of spacer length
}

\author{
Mohamad Yusuf, ${ }^{* a}$ Rupesh Kumar, ${ }^{a}$ and S. C. Gupta ${ }^{\text {b }}$ \\ ${ }^{a}$ Department of Chemistry, Punjabi University, Patiala, Punjab 147002, India \\ ${ }^{b}$ Chemistry Department, Kurukshetra University, Kurukshetra, Haryana 116119, India \\ E-mail: dryusufpup@,rediffmail.com
}

\begin{abstract}
Photocyclizations of 1,10-decylbischromones occurring through intramolecular H-abstractions involving 1,4-biradicals have been described. Here, the length of the extended spacer unit has affected the yield and distribution of the photoproducts.
\end{abstract}

Keywords: Chromophore, cyclization, spacer, decylbischromones, 1,4-biradical

\section{Introduction}

Bischromones are the bichromophoric substrates that are formed by joining the two chromone nuclei together through a carbon chain of varying length and structure. These molecules do not exhibit any interactions among chromophores in the ground state, but these interactions become operative under the influence of light to generate excited state complexes that lead to many deactivation processes as well as formation of photoproducts. ${ }^{1-3}$ Photoexcited bisanthracenes, ${ }^{4,5}$ biscoumarins, ${ }^{6,7}$ bispyrones $^{8}$ and bisalkoxybenzophenones ${ }^{9}$ suffered such intramolecular bichromophoric interactions. Here, the photoproduct formation was affected by the nature of the spacer linking the two chromophores.

We have reported recently the phototransformations of some 3 -alkoxy ${ }^{10}$ as well as xylylbischromonenes ${ }^{11,12}$ that provided cyclised products depending upon the length and rigidity of the intervening carbon bridges. These results have prompted us further to explore photocyclisations upon the bischromones 1 and $\mathbf{2}$ with the well-separated chromophoric units, built around a decyl moiety. The main impetus behind this study was to investigate the affect of the lengthy and flexible decyl spacer upon the product formation/distribution in the photoreactions. 


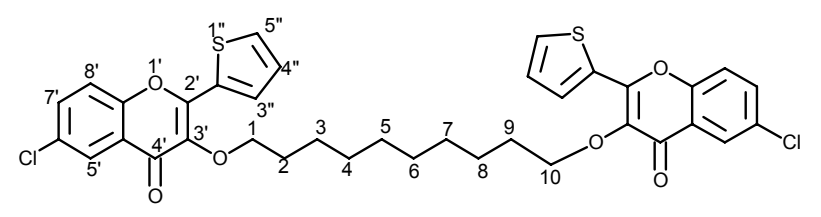

1

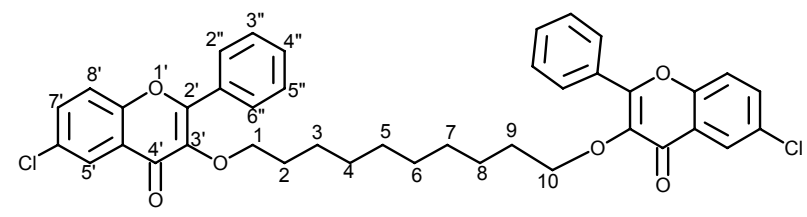

2

Figure 1.

\section{Results and Discussion}

The bischromones 1 and 2 needed for this study were synthesized by reacting 6-chloro-3hydroxy-2-(2'-thienyl)-4-oxo-4H-1-benzopyran ${ }^{13}$ and 6-chloro-3-hydroxy-2-phenyl-4-oxo-4H-1benzopyran, ${ }^{14}$ respectively, with 1,10-dibromodecane in the presence of dry $\mathrm{K}_{2} \mathrm{CO}_{3} /$ Acetone and $\mathrm{Bu}_{4} \mathrm{~N}^{+} \mathrm{I}^{-}$as PTC, which not only decreased the reaction time drastically but also improved the yield of bischromones. The monoalkylated chromones formed in the reaction mixtures were removed by column chromatography.

The structures of bischromones $\mathbf{1}$ and $\mathbf{2}$ were analyzed from the rigorous analysis of their IR / ${ }^{1} \mathrm{H}$ NMR spectra (see experimental). The photoirradiation of a deoxygenated benzene solution of 1 with light from a $125 \mathrm{~W}$ mercury arc lamp yielded four photoproducts 3-6. Similarly, further photolysis of $\mathbf{2}$ in benzene solution furnished three photoproducts 7-9. The structures of these photoproducts were confirmed by comparison of their IR / ${ }^{1} \mathrm{H}$ NMR spectra with those of $\mathbf{1}$ and $\mathbf{2}$ (see experimental) and the similar photoproducts obtained from the photoirradiation of bischromones ${ }^{10} \mathbf{1 1}$ and $\mathbf{1 2 .}$

IR spectra of these photoproducts proved very helpful to predict the involvement of either one or both chromophoric units during the phototransformations. The photoproducts $\mathbf{3}$ and $\mathbf{4}$ are formed through the involvement of only one of the two chromophores as revealed by two IR absorptions (1635, 1653 and $1631,1649 \mathrm{~cm}^{-1}$, respectively) in the carbonyl region and the IR spectra of 5 and 6 exhibited only one intense absorption (1651 and $1648 \mathrm{~cm}^{-1}$ respectively) in their carbonyl region, indicating the involvement of both chromophoric units in the photoreaction. Similarly, photoproducts $7\left(1633,1659 \mathrm{~cm}^{-1}\right)$ and $\mathbf{8}\left(1635,1644 \mathrm{~cm}^{-1}\right)$ could arise from the reaction of only one chromophoric unit while both chromophores underwent photoreaction to provide product $9\left(1643 \mathrm{~cm}^{-1}\right)$. These observations were lent further support from their $300 \mathrm{MHz}{ }^{1} \mathrm{H}$ NMR spectra (see experimental). 


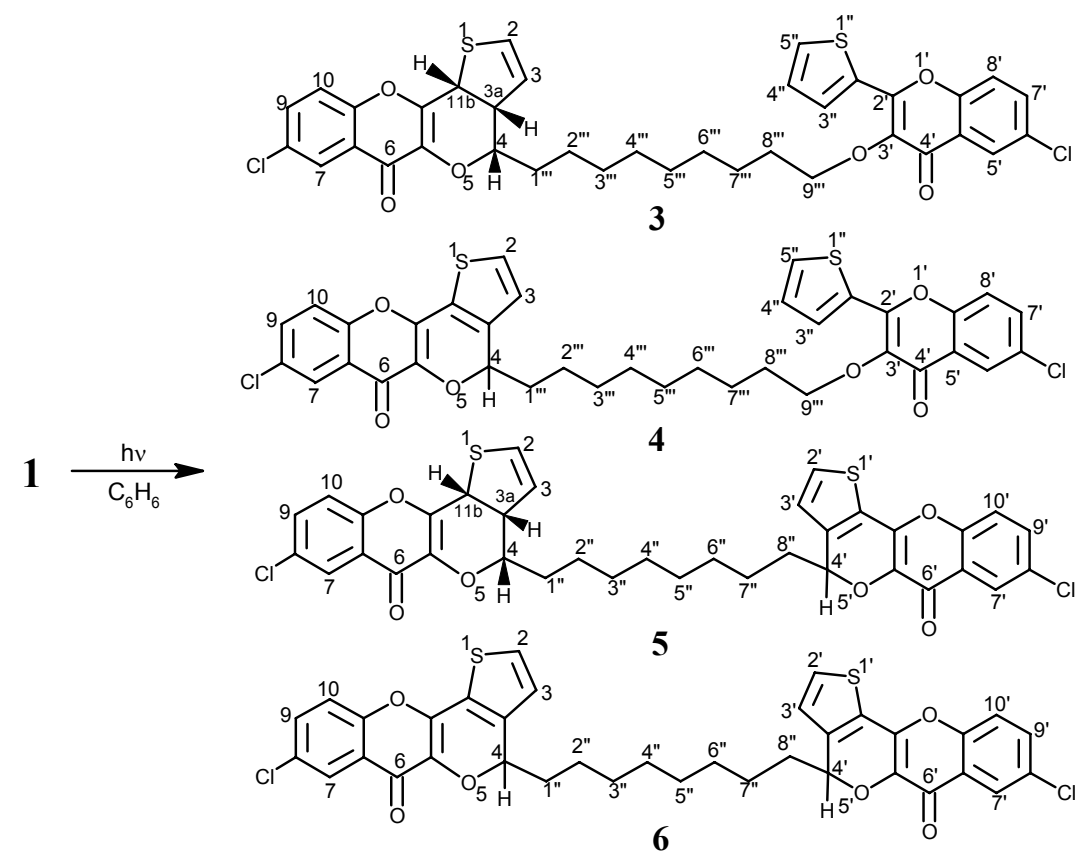

\section{Scheme 1}

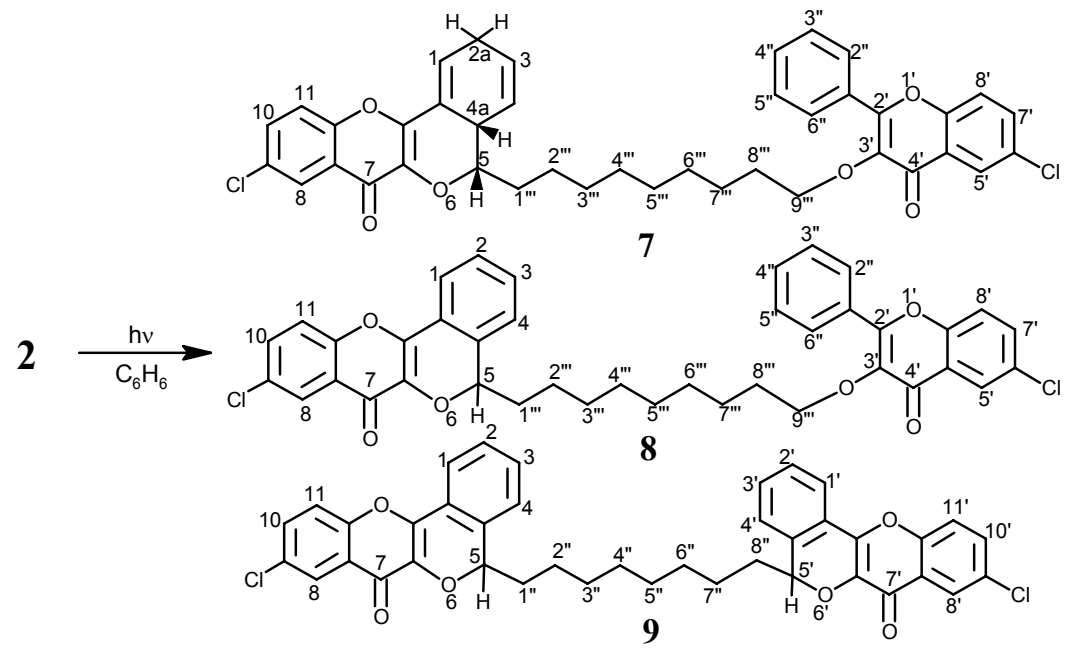

\section{Scheme 2}

The stereochemical features of hydrogens at C-3a, C-4 and C-11b in photoproducts 5 and 7 were ascertained from $J$ and $\Phi$ parameters: that all the hydrogens are present on the same side is evident from $J_{3 a, 4}=11 \mathrm{~Hz}\left(\Phi=0^{\circ}\right)$ and $J_{11 b, 3 a}=8.5 \mathrm{~Hz}\left(\Phi \sim 27^{\circ}\right){ }^{13,15,16}$ The heavier alkyl group at $\mathrm{C}-4$ is present in the preferred $\Psi$-equatorial conformation and ring $\mathrm{C}$ in the half chair form. ${ }^{11}$

Mechanistically, the photoreorganisations of $\mathbf{1}$ and $\mathbf{2}$ can be visualized as having occurred through an initial $\mathrm{H}$-abstraction from the $-\mathrm{OCH}_{2}$ - group by the photoexcited carbonyl chromophore of the pyrone moiety to produce 1,4-biradical 10 (Figure 2) that further leads to cyclization $^{11,14,15}$ to yield various cyclized and dehydrogenated cyclized photoproducts. Even the 
solvent polarity $\left(\mathrm{C}_{6} \mathrm{H}_{6}, \mathrm{MeOH}\right.$ and THF $)$ did not have any significant influence on the product formation/distribution. This again supports that these reorganizations occurred only through intramolecular $\mathrm{H}$-abstractions.

The aromatic compounds $\mathbf{4}$ and $\mathbf{8}$ are not obtained from the photodehydrogenation of dihydro compounds 3 and 7, but are formed independently from the common 1,4-biradical intermediate 10 (Scheme 5). This phenomenon was corroborated from the fact that further photoirradiation of dihydro compounds $\mathbf{3}$ and 7, did not give any aromatic compound but yielded only some polymeric material.

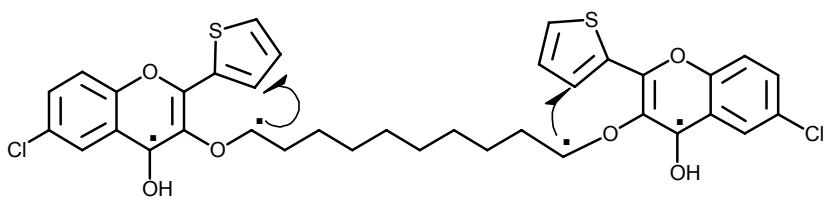

10

Figure 2

In the photolysis of bischromone 11, in addition to photoproducts 3-6, there could be another product where both thienyl rings are dihydro. Also the photoirradiation of $\mathbf{2}$, in addition to the product 7-9, could have furnished two more products, one similar to $\mathbf{5}$ where one phenyl ring is aromatic and the other is dihydro, and other product where both phenyl rings are dihydro. The possibility of the formation of such photoproducts cannot be excluded completely, as these products may be formed in very low yield such that their isolation was unsuccessful.

The photolysis of bischromones $\mathbf{1}$ and $\mathbf{2}$ resulted in better chemical efficiency in terms of photoproduct formation and distribution, in comparison to similar irradiation of bischromones ${ }^{10}$ 11 and 12.

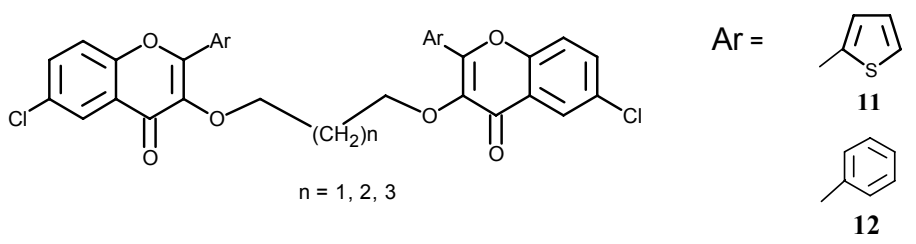

Figure 3

Table 1. Photolytic conversion of bischromones

\begin{tabular}{ccccc}
\hline Bischromones & \multicolumn{4}{c}{ Total photolytic conversion } \\
\cline { 2 - 5 } & $\mathbf{n = 8}$ & $\mathbf{n}=\mathbf{3}^{10}$ & $\mathbf{n}=\mathbf{2}^{10}$ & $\mathbf{n}=\mathbf{1}^{10}$ \\
\hline $\mathbf{1 1}$ & 40 & 31 & 22 & 25 \\
$\mathbf{1 2}$ & 45 & 29 & 30 & 30 \\
\hline
\end{tabular}


It is evident from Table 1 that the introduction of the lengthy intermediate bridge (decyl moiety) in bischromone $1(n=8)$ and $2(n=8)$ has been instrumental in furnishing total photolytic conversion to an extent about one and half times greater than for bischromones 11 (n $=1,2,3)$ and $12(n=1,2,3)$. Probably, this phenomenon can be ascribed to the ease of formation of the excited state intramolecular complexes in the bischromones with shorter spacers $(\mathrm{n}=2,1)$. Thus, as the distance increases among the chromophores, then the probability of intramolecular complex formation decreases, minimizing the involvement of deactivation processes that causes the improved yields and distributions of photoproducts. In these cases, the photoproduct from the intramolecular [2+2] cycloaddition of both pyrone rings was not realized. However, intermolecular [2+2] photocycloadditions of benzo- $\gamma$-pyrone have been well established. ${ }^{17}$

It may be concluded that the inclusion of a flexible, lengthy spacer in bischromones $\mathbf{1}$ and 2 makes both photoexcited chromophores liable to behave independently and induces better chemical efficiency in the photoreorganisation. It is worth mentioning here that these photolytic routes present a versatile and efficient tool for the generation of tetracyclic bischromones that are very difficult to prepare under the usual thermal modes.

\section{Experimental Section}

General Procedures. Melting points reported are uncorrected. IR spectra were recorded on a Buck Scientific 500 Spectrophotometer using KBr pellets and UV spectra on U-2000 HitachiSpectrophotometer. ${ }^{1} \mathrm{H}$ NMR spectra were recorded on a $300 \mathrm{MHz}$ Bruker spectrometer using TMS as internal standard. TLC plates were coated with silica gel G suspended in $\mathrm{MeOH}-\mathrm{CHCl}_{3}$. Silica gel (100-200 mesh) was used for column chromatography. The percent yields reported in the photochemical reactions are calculated by excluding the recovered starting materials.

Preparation of 1,10-bis[6'-chloro-2'-(2"'-thienyl)-4'-oxo-4'H-1'-benzopyran-3'-oxy]decane (1). General procedure

A suspension of 6-chloro-3-hydroxy-2-(2'-thienyl)-4-oxo-4H-1-benzopyran ${ }^{13}$ (2.8 g, $\left.0.01 \mathrm{~mol}\right)$, 1,10-dibromodecane $(1.5 \mathrm{~g}, 0.005 \mathrm{~mol})$, tetrabutylammonium iodide $(2.0 \mathrm{~g})$ and freshly ignited $\mathrm{K}_{2} \mathrm{CO}_{3}(1.0 \mathrm{~g})$ was refluxed in dry acetone $(25 \mathrm{ml})$ for $1 \mathrm{~h}$ with stirring. The color of the reaction mixture changed from deep red to colorless. The reaction mixture after filtration and the distillation of acetone was poured into iced $\mathrm{HCl}$ to obtain a mixture of monochromone and bischromone. This mixture was then percolated through a column of silica-gel (100-200 mesh) using benzene as eluent and crystallized from $\mathrm{CHCl}_{3}-\mathrm{MeOH}(1: 2)$ mixture to furnish pure bischromone 1 .

Yield: $50 \%$ of colourless solid: $\mathrm{mp} 170-172{ }^{\circ} \mathrm{C}$; UV (THF) $\lambda_{\max }(\varepsilon): 344 \mathrm{~nm}(17700), 328 \mathrm{~nm}$ (18100), $260 \mathrm{~nm}$ (14700); IR (KBr) $v_{\max } / \mathrm{cm}^{-1}: 1632(\mathrm{C}=\mathrm{O}) ;{ }^{1} \mathrm{H} \mathrm{NMR}\left(\mathrm{CDCl}_{3}, 300 \mathrm{MHz}\right)$

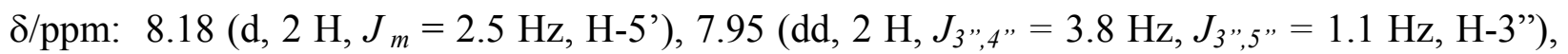
$7.64\left(\mathrm{dd}, 2 \mathrm{H}, J_{5}\right.$ ", ${ }^{\prime}=5.0 \mathrm{~Hz}, J_{5}$ ", $3^{\prime}=1.1 \mathrm{~Hz}, \mathrm{H}-5$ "), 7.58 (dd, $\left.2 \mathrm{H}, J_{m, o}=2.5 \mathrm{~Hz}, 8.9 \mathrm{~Hz}, \mathrm{H}-7^{\prime}\right)$, 
7.48 (d, $\left.2 \mathrm{H}, J_{o}=8.9 \mathrm{~Hz}, \mathrm{H}-8^{\prime}\right), 7.21$ (dd, $2 \mathrm{H}, J_{4}$ ", 3" = $3.8 \mathrm{~Hz}, J_{4}$ ", 5 " = $5.0 \mathrm{~Hz}, \mathrm{H}-4$ "), 4.26 (t, 4 $\left.\mathrm{H}, J_{\text {vic }}=7.0 \mathrm{~Hz}, \mathrm{H}-1,10\right), 1.93$ (q, $\left.4 \mathrm{H}, J_{\text {vic }}=7.1 \mathrm{~Hz}, \mathrm{H}-2,9\right), 1.48$ (m, $\left.12 \mathrm{H}, \mathrm{H}-3,4,5,6,7,8\right)$.

\section{Preparation of 1,10-bis[6'-chloro-2'-phenyl-4'-oxo-4'H-1'-benzopyran-3'-oxy]decane (2)}

The bischromones 2 was obtained by the alkylation of 6-chloro-3-hydroxy-2-phenyl-4-oxo- $4 \mathrm{H}$ 1-benzopyran ${ }^{14}(2.7 \mathrm{~g}, 0.01 \mathrm{~mol})$ with 1,10-dibromodecane $(1.5 \mathrm{~g}, 0.005 \mathrm{~mol})$, according to the same procedure as used for 1 .

Yield: $60 \%$ of colourless solid: $\mathrm{mp} 136-138{ }^{\circ} \mathrm{C}$; UV (THF) $\lambda_{\max }(\varepsilon): 302 \mathrm{~nm}(13400), 246 \mathrm{~nm}$ (18400); IR (KBr) $v_{\max } / \mathrm{cm}^{-1} 1635(\mathrm{C}=\mathrm{O}) ;{ }^{1} \mathrm{H} \mathrm{NMR}\left(\mathrm{CDCl}_{3}, 300 \mathrm{MHz}\right) \delta / \mathrm{ppm} 8.22\left(\mathrm{~d}, 2 \mathrm{H}, J_{m}\right.$ = $2.6 \mathrm{~Hz}, \mathrm{H}-5$ '), 8.07 (m, $4 \mathrm{H}, \mathrm{H}-2$ ", 6”), 7.60 (dd, 2 H, $J_{m, o}=2.6 \mathrm{~Hz}, 8.9 \mathrm{~Hz}, \mathrm{H}-7$ '), 7.50 (m, 6 H, H-3",4",5"), 7.48 (d, 2 H, Jo = 8.9 Hz, H-8'), 4.03 (t, 4 H, $\left.J_{v i c}=6.0 \mathrm{~Hz}, \mathrm{H}-1,10\right), 1.32$ (m, 4 H, H-2,9), 1.28 (m, 4 H, H-3,8), 1.25 (m, 8 H, H-4,5,6,7).

Photolysis of 1,10-bis[6'-chloro-2'-(2''-thienyl)-4'-oxo-4'H-1'-benzopyran-3'-oxy]decane (1). General procedure

A benzene solution $(100 \mathrm{~mL})$ of $1(100 \mathrm{mg}, 0.00014 \mathrm{~mol})$ was irradiated with the light of $125 \mathrm{~W}$ mercury arc lamp in a pyrex reactor under nitrogen atmosphere for $45 \mathrm{~min}$. The progress of the reaction was monitored by tlc (benzene-EtOAc, 9:1). The reaction mixture developed an intense yellow color during the course of photoreaction. Even the photolysis for longer duration did not show the complete disappearance of the starting compound. The solvent was distilled under reduced pressure and the photolysate was chromatographed over a column of silica-gel (100-200 mesh) packed in benzene. Elution of column with benzene-EtOAc (8:2) yielded starting compound 1 (20\%, co-tlc and mp) and four new compounds 3-6.

Compound 3. Yield: $10 \%$ of pale yellow solid: $\mathrm{mp} 150-153{ }^{\circ} \mathrm{C}$; UV (THF): $\lambda_{\max }(\varepsilon): 325 \mathrm{~nm}$ (16000), $260 \mathrm{~nm}$ (16500); IR (KBr) $v_{\max } / \mathrm{cm}^{-1}: 1653(\mathrm{C}=\mathrm{O}), 1635(\mathrm{C}=\mathrm{O}) ;{ }^{1} \mathrm{H}$ NMR $\left(\mathrm{CDCl}_{3}, 300\right.$

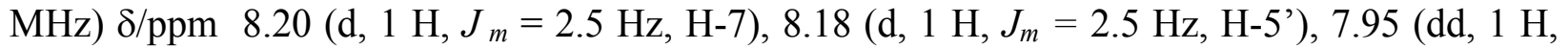

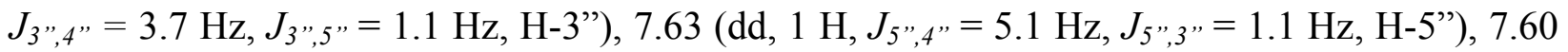
$\left(\mathrm{dd}, 1 \mathrm{H}, J_{m, o}=2.5 \mathrm{~Hz}, 8.9 \mathrm{~Hz}, \mathrm{H}-9\right), 7.58\left(\mathrm{dd}, 1 \mathrm{H}, J_{m, o}=2.4 \mathrm{~Hz}, 8.9 \mathrm{~Hz}, \mathrm{H}-7\right.$ ') $7.48\left(\mathrm{~d}, 1 \mathrm{H}, J_{o}\right.$ $\left.=8.9 \mathrm{~Hz}, \mathrm{H}-8^{\prime}\right), 7.39$ (d, $\left.1 \mathrm{H}, J_{o}=8.9 \mathrm{~Hz}, \mathrm{H}-10\right), 7.21\left(\mathrm{dd}, 1 \mathrm{H}, J_{4}{ }^{\prime \prime},{ }^{\prime}=5.1 \mathrm{~Hz}, J_{4}\right.$ ", $3^{\prime \prime}=3.7 \mathrm{~Hz}$, H-4"), 6.38 (dd, $\left.1 \mathrm{H}, J_{2,3}=5.9 \mathrm{~Hz}, J_{2,3 a}=1.0 \mathrm{~Hz}, \mathrm{H}-2\right), 5.62\left(\mathrm{dd}, 1 \mathrm{H}, J_{3,2}=5.9 \mathrm{~Hz}, J_{3,3 a}=3.3\right.$ Hz, H-3), 4.95 (d, $\left.1 \mathrm{H}, J_{11 b, 3 a}=8.5 \mathrm{~Hz}, \mathrm{H}-11 \mathrm{~b}\right), 4.24$ (t, $2 \mathrm{H}, J_{\text {vic }}=6.4 \mathrm{~Hz}, \mathrm{H}-9$ '”), 3.90 (dd, $1 \mathrm{H}$, $\left.J_{4,3 a}=11.0 \mathrm{~Hz}, J_{\text {vic }}=6.0 \mathrm{~Hz}, \mathrm{H}-4\right), 3.21\left(\mathrm{ddd}, 1 \mathrm{H}, J_{3 a, 3}=3.3 \mathrm{~Hz}, J_{3 a, 11 b}=8.5 \mathrm{~Hz}, J_{3 a, 4}=11.0 \mathrm{~Hz}\right.$, H-3a), 1.75 (m, 4 H, H-1"',8"'), 1.33 (m, 12 H, H-2"',3"',4"',5",,6",,7"'); MS m/z (\%): 695 (100.0) [MH] ${ }^{+}, 694$ (43.62), 693 (11.24), 499 (14.94), 418 (7.6), 417 (15.67), 291 (1.47), 290 (1.0), 281 (6.54), 278 (11.40), 277 (2.40), 263 (4.85), 155 (1.30), 154 (2.31), 136 (5.36); Anal. Calcd for $\mathrm{C}_{36} \mathrm{H}_{32} \mathrm{O}_{6} \mathrm{Cl}_{2} \mathrm{~S}_{2}$ : C 62.16, $\mathrm{H}$ 4.60. Found: $\mathrm{C} 62.25, \mathrm{H} 4.51$.

Compound 4. Yield: $20 \%$ of pale yellow solid: mp 161-163 ${ }^{\circ} \mathrm{C}$; UV (THF) $\lambda_{\max }(\varepsilon): 352 \mathrm{~nm}$ (15300), $324 \mathrm{~nm}$ (15200), $260 \mathrm{~nm}$ (16000); IR (KBr) $v_{\max } / \mathrm{cm}^{-1}: 1649(\mathrm{C}=\mathrm{O}), 1631(\mathrm{C}=\mathrm{O}) ;{ }^{1} \mathrm{H}$ NMR $\left(\mathrm{CDCl}_{3}, 300 \mathrm{MHz}\right) \delta / \mathrm{ppm} 8.26\left(\mathrm{~d}, 1 \mathrm{H}, J_{m}=2.4 \mathrm{~Hz}, \mathrm{H}-7\right), 8.17\left(\mathrm{~d}, 1 \mathrm{H}, J_{m}=2.4 \mathrm{~Hz}, \mathrm{H}-\right.$ 5'), 7.95 (dd, $1 \mathrm{H}, J_{3}$ ", 4" = $3.6 \mathrm{~Hz}, J_{3}$ ", 5" = $1.1 \mathrm{~Hz}, \mathrm{H}-3$ "), 7.63 (dd, $1 \mathrm{H}, J_{5}$ ", 4" = $5.0 \mathrm{~Hz}, J_{5}$ ", 3" = $1.1 \mathrm{~Hz}, \mathrm{H}-5$ "), 7.60 (dd, $\left.1 \mathrm{H}, J_{m, o}=2.4 \mathrm{~Hz}, 8.9 \mathrm{~Hz}, \mathrm{H}-9\right), 7.58$ (dd, $1 \mathrm{H}, J_{m, o}=2.4 \mathrm{~Hz}, 8.9 \mathrm{~Hz}$, H-7'), 7.48 (d, 1 H, $\left.J_{o}=8.9 \mathrm{~Hz}, \mathrm{H}-10\right), 7.45$ (d, $1 \mathrm{H}, J_{o}=8.9 \mathrm{~Hz}, \mathrm{H}-8$ '), 7.21 (d, $1 \mathrm{H}, J=4.8$ 
Hz, H-2,4”), 6.93 (d, $\left.1 \mathrm{H}, J_{3,2}=4.8 \mathrm{~Hz}, \mathrm{H}-3\right), 5.55$ (dd, $\left.1 \mathrm{H}, J=5.1 \mathrm{~Hz}, 7.0 \mathrm{~Hz}, \mathrm{H}-4\right), 4.25$ (t, 2 H, $J_{v i c}=6.9 \mathrm{~Hz}, \mathrm{H}-9$ "'), 2.10 (m, $2 \mathrm{H}, \mathrm{H}-1$ "”), 1.95 (m, $2 \mathrm{H}, \mathrm{H}-8$ "”), 1.45 (m, $12 \mathrm{H}, \mathrm{H}-$ 2",,3"',4", 5", ,6", 7"”); MS m/z (\%): 693 (100.0) [MH] ${ }^{+}, 692$ (38.07), 416 (5.0), 291 (4.97), 279 (1.48), 278 (2.77), 263 (1.39), 186 (2.50), 183 (1.07), 169 (1.00); Anal. Calcd for $\mathrm{C}_{36} \mathrm{H}_{30} \mathrm{O}_{6} \mathrm{Cl}_{2} \mathrm{~S}_{2}$ : C 62.34, H 4.33. Found C 62.25, H 4.40.

Compound 5. Yield: $5 \%$ of pale yellow solid: $\mathrm{mp} 172-174{ }^{\circ} \mathrm{C}$; UV (THF) $\lambda_{\max }(\varepsilon): 388 \mathrm{~nm}$ (14800), $369 \mathrm{~nm}$ (26000), $323 \mathrm{~nm}$ (25000); IR (KBr) $v_{\max } / \mathrm{cm}^{-1}: 1651(\mathrm{C}=\mathrm{O}) ;{ }^{1} \mathrm{H} \mathrm{NMR}\left(\mathrm{CDCl}_{3}\right.$, $300 \mathrm{MHz}$ ) $\delta / \mathrm{ppm}: 8.26\left(\mathrm{~d}, 1 \mathrm{H}, J_{m}=2.4 \mathrm{~Hz}, \mathrm{H}-7\right), 8.20$ (d, $1 \mathrm{H}, J_{m}=2.4 \mathrm{~Hz}, \mathrm{H}-7$ '), 7.60 (d, 1 $\left.\mathrm{H}, J_{m, o}=2.4 \mathrm{~Hz}, 8.9 \mathrm{~Hz}, \mathrm{H}-9\right), 7.59$ (dd, $1 \mathrm{H}, J_{m, o}=2.4 \mathrm{~Hz}, 8.9 \mathrm{~Hz}, \mathrm{H}-9$ ') 7.48 (d, $1 \mathrm{H}, J_{o}=8.9$ Hz, H-10), 7.39 (d, 1 H, Jo = 8.9 Hz, H-10'), 7.20 (d, 1 H, $J_{2}^{\prime}, 3$ ' = 4.8 Hz, H-2'), 6.94 (d, 1 H, $\left.J_{3^{\prime}, 2}{ }^{\prime}=4.8 \mathrm{~Hz}, \mathrm{H}-3^{\prime}\right), 6.38\left(\mathrm{~d}, 1 \mathrm{H}, J_{2,3}=6.0 \mathrm{~Hz}, \mathrm{H}-2\right), 5.62\left(\mathrm{dd}, 1 \mathrm{H}, J_{3,3 a}=3.0 \mathrm{~Hz}, J_{3,2}=6.0 \mathrm{~Hz}\right.$, H-3), 5.50 (dd, $1 \mathrm{H}, J=5.1 \mathrm{~Hz}, 6.9 \mathrm{~Hz}, \mathrm{H}-4$ '), 4.96 (d, $\left.1 \mathrm{H}, J_{11 b, 3 a}=8.5 \mathrm{~Hz}, \mathrm{H}-11 \mathrm{~b}\right), 3.90$ (m, 1 H, H-4), 3.21 (ddd, $\left.1 \mathrm{H}, J_{3 a, 3}=3.0 \mathrm{~Hz}, J_{3 a, 11 b}=8.5 \mathrm{~Hz}, J_{3 a, 4}=11.0, \mathrm{H}-3 \mathrm{a}\right), 2.01$ (m, $2 \mathrm{H}, \mathrm{H}-8^{\prime}$ ), 1.95 (m, 2 H, H-1"), 1.28 (m, 12 H, H-2",3",4”,5”,6”,7”); MS m/z (\%): 693 (100.0) [MH] $]^{+}, 692$ (29.65), 691 (42.19), 690 (29.65), 489 (8.77), 432 (7.47), 418 (2.70), 403 (2.67), 304 (13.89), 291 (4.40), 278 (5.55), 264 (4.59), 262 (11.41); Anal. Calcd for $\mathrm{C}_{36} \mathrm{H}_{30} \mathrm{O}_{6} \mathrm{Cl}_{2} \mathrm{~S}_{2}: \mathrm{C}$ 62.34, H 4.33. Found C 62.21, H 4.42 .

Compound 6. Yield: $5 \%$ of pale yellow solid: mp $185-187{ }^{\circ} \mathrm{C}$; UV (THF) $\lambda_{\max }(\varepsilon): 354 \mathrm{~nm}$ (15900), $324 \mathrm{~nm}$ (19000); IR (KBr) v $v_{\max } / \mathrm{cm}^{-1}: 1648$ (C=O); ${ }^{1} \mathrm{H}$ NMR $\left(\mathrm{CDCl}_{3}, 300 \mathrm{MHz}\right) \delta / \mathrm{ppm}$ : $8.26\left(\mathrm{~d}, 2 \mathrm{H}, J_{m}=2.4 \mathrm{~Hz}, \mathrm{H}-7,7^{\prime}\right), 7.60$ (d, $2 \mathrm{H}, J_{m, o}=2.4 \mathrm{~Hz}, 9.0 \mathrm{~Hz}, \mathrm{H}-9,9$ ') 7.47 (d, $2 \mathrm{H}, J_{o}=$ $9.0 \mathrm{~Hz}, \mathrm{H}-10,10^{\prime}$ ), 7.21 (d, $2 \mathrm{H}, J_{2,3}=4.8 \mathrm{~Hz}, \mathrm{H}-2,2^{\prime}$ ), 6.93 (d, $\left.2 \mathrm{H}, J_{3,2}=4.8 \mathrm{~Hz}, \mathrm{H}-3,3^{\prime}\right), 5.50$ (dd, 2 H, $J=5.1 \mathrm{~Hz}, 6.9 \mathrm{~Hz}, \mathrm{H}-4,4$ '), 2.10 (m, 4 H, H-1",8”), 1.55 (m, 12 H-2",3",4",5",6",7”); MS $m / z$ (\%): 691 (100.0) [MH] ${ }^{+}, 690$ (39.37), 689 (10.96), 688 (16.92), 434 (19.10), 432 (27.22), 354 (14.21), 305 (3.49), 291 (2.27), 290 (23.77), 263 (9.97), 204 (60.32), 236 (22.63); Anal. Calcd for $\mathrm{C}_{36} \mathrm{H}_{28} \mathrm{O}_{6} \mathrm{Cl}_{2} \mathrm{~S}_{2}$ : C 62.52, $\mathrm{H}$ 4.05. Found C 62.43, H 4.13.

\section{Photolysis of 1,10-bis[6'-chloro-2'-phenyl-4'-oxo-4'H-1'-benzopyran-3'-oxy]decane (2)}

A deoxygenated benzene solution $(100 \mathrm{ml})$ of $2(100 \mathrm{mg}, 0.00015 \mathrm{~mol})$ was photoirradiated and resulting photoproducts 7-9 were separated according to the same procedure followed for the photolysis of $\mathbf{1}$.

Compound 7. Yield: 5\% of pale yellow solid: mp 126-128 ${ }^{\circ} \mathrm{C}$; UV (THF) $\lambda_{\max }(\varepsilon): 315 \mathrm{~nm}$ (16000), $246 \mathrm{~nm}$ (20000); IR (KBr) v $v_{\max } / \mathrm{cm}^{-1}: 1659(\mathrm{C}=\mathrm{O}), 1633(\mathrm{C}=\mathrm{O}) ;{ }^{1} \mathrm{H} \mathrm{NMR}\left(\mathrm{CDCl}_{3}, 300\right.$

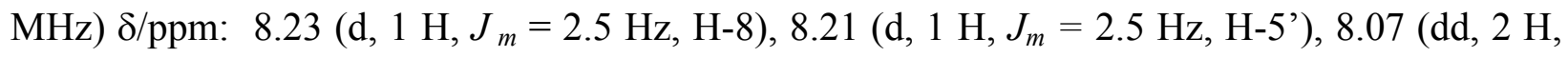
$J_{m, o}=2.1 \mathrm{~Hz}, 7.8 \mathrm{~Hz}, \mathrm{H}-2$ "',6”), 7.61 (dd, $2 \mathrm{H}, J_{m, o}=2.5 \mathrm{~Hz}, 8.9 \mathrm{~Hz}, \mathrm{H}-10,7$ '), 7.51 (m, $3 \mathrm{H}, \mathrm{H}-$ 3",4",5”), 7.38 (d, 2 H, Jo = 8.9 Hz, H-11,8'), 6.67 (d, 1 H, $J_{1,2 a}=2.0 \mathrm{~Hz}, \mathrm{H}-1$ ), 5.96 (dd, $1 \mathrm{H}$, $\left.J_{3,2 a}=2.0 \mathrm{~Hz}, J_{3,4}=11.8 \mathrm{~Hz}, \mathrm{H}-3\right), 5.75\left(\mathrm{~d}, 1 \mathrm{H}, J_{4,3}=11.8 \mathrm{~Hz}, \mathrm{H}-4\right), 4.13$ (m, $2 \mathrm{H}, \mathrm{H}-9$ '”), 3.77 (m, $1 \mathrm{H}, \mathrm{H}-5), 3.30$ (m, $1 \mathrm{H}, \mathrm{H}-4 \mathrm{a}), 2.90$ (br s, $2 \mathrm{H}, \mathrm{H}-2 \mathrm{a}), 2.20$ (m, $4 \mathrm{H}, \mathrm{H}-1$ "', ,8"'), 1.95 (m, 12 H, H-2"',3", ,4", 5" ,6", ,7"'); MS m/z (\%): 682 (2.5) [M] $]^{+}, 412$ (10.90), 411 (10.90), 410 (8.94), 284 (14.82), 272 (95.76), 271 (100.0), 256 (14.51), 243 (5.20), 215 (9.19), 155 (5.50), 154 (9.44), 118 (3.0); Anal. Calcd for $\mathrm{C}_{40} \mathrm{H}_{36} \mathrm{O}_{6} \mathrm{Cl}_{2}$ : C 70.28, H 5.27. Found: C 70.36, H 5.20. 
Compound 8. Yield: $25 \%$ of pale yellow solid: mp 140-142 ${ }^{\circ} \mathrm{C}$; UV (THF) $\lambda_{\max }(\varepsilon): 315 \mathrm{~nm}$ (16900), 306 nm (15200); IR (KBr) $v_{\max } / \mathrm{cm}^{-1}: 1644(\mathrm{C}=\mathrm{O}), 1635(\mathrm{C}=\mathrm{O}) ;{ }^{1} \mathrm{H}$ NMR $\left(\mathrm{CDCl}_{3}, 300\right.$

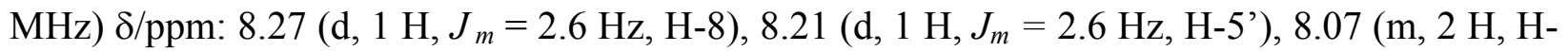
2",6”), 7.86 (dd, $1 \mathrm{H}, J=2.5 \mathrm{~Hz}, 8.0 \mathrm{~Hz}, \mathrm{H}-1$ ), 7.61 (dd, $\left.1 \mathrm{H}, J_{m, o}=2.6 \mathrm{~Hz}, 8.9 \mathrm{~Hz}, \mathrm{H}-10\right), 7.60$ (dd, $1 \mathrm{H}, J_{m, o}=2.6 \mathrm{~Hz}, 8.9 \mathrm{~Hz}, \mathrm{H}-7$ '), 7.49 (m, $7 \mathrm{H}, \mathrm{H}-2,3,11,8$ ',3”, 4",5”), 7.16 (dd, $1 \mathrm{H}, J=2.4$ Hz, 6.0 Hz, H-4), 5.34 (dd, 1 H, J=5.0 Hz, 8.1 Hz, H-5), 4.01 (t, 2 H, Jvic $=6.6$ Hz, H-9'”), 2.05 (m, 2 H, H-1"'), 1.25 (m, 14 H, H-2" ,3"',4", 5" ,6", 7", ,8" ); MS m/z (\%): 680 (16.23) [M] ${ }^{+}$, 409 (18.32), 284 (22.54), 283 (96.79), 272 (100.0), 271 (69.22), 243 (5.66), 215 (11.55), 155 (25.69), 154 (15.54), 126 (7.45), 118 (4.86), 110 (5.0), 105 (23.05), 77 (22.17); Anal. Calcd for $\mathrm{C}_{40} \mathrm{H}_{34} \mathrm{O}_{6} \mathrm{Cl}_{2}$ : C 70.48, $\mathrm{H} 4.98$. Found: $\mathrm{C} 70.35, \mathrm{H} 4.91$.

Compound 9. Yield: $15 \%$ of pale yellow solid: mp $170-72{ }^{\circ} \mathrm{C}$; UV (THF) $\lambda_{\max }(\varepsilon): 352 \mathrm{~nm}$ (17600), $316 \mathrm{~nm}$ (18300), 247 (24500); IR (KBr) $v_{\max } / \mathrm{cm}^{-1}: 1643$ (C=O); ${ }^{1} \mathrm{H} \mathrm{NMR}\left(\mathrm{CDCl}_{3}, 300\right.$

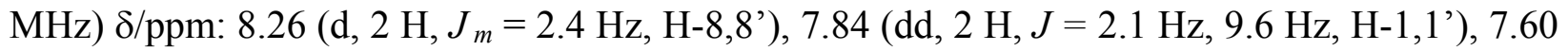
(dd, 2 H, $J_{m, o}=2.4 \mathrm{~Hz}, 9.0 \mathrm{~Hz}, \mathrm{H}-10,10$ ') 7.52 (d, 2 H, $\left.J_{o}=9.0 \mathrm{~Hz}, \mathrm{H}-11,11^{\prime}\right), 7.44$ (m, 4 H, H2,3,2',3'), 7.17 (dd, 2 H, J=2.2 Hz, 6.0 Hz, H-4,4'), 5.32 (dd, 2 H, J=5.1 Hz, 8.4 Hz, H-5,5'), 2.0 (m, 4 H, H-1",8”), 1.25 (m, 12 H, H-2",3",4",5",6”,7”); MS m/z (\%): 678 (25.0) [M] 312 (3.24), 311 (11.66), 285 (37.50), 284 (25.17), 283 (100.0), 272 (10.45), 271 (8.29), 155 (23.89), 154 (5.3), 129 (5.40); Anal. Calcd for $\mathrm{C}_{40} \mathrm{H}_{32} \mathrm{O}_{6} \mathrm{Cl}_{2}$ : C 70.69, H 4.71. Found: $\mathrm{C}$ 70.60, H 4.78.

\section{Acknowledgement}

Author is highly thankful to CSIR, N. Delhi, India for the generous grant. The necessary facilities and some financial assistance provided by Prof. N.R. Dhamiwal, Head, Department of Chemistry, Punjabi University, Patiala, has also been highly acknowledged.

\section{References}

1. Stevens, B. Nature 1962, 192.

2. Forster, T. Angew. Chem. Int. Ed. 1969, 8, 333.

3. Birks, J. B. Progress in Reaction Kinetics, Porter, G. Ed.; Pergamon: New York, 1971; Vol. $5, \mathrm{p} 181$.

4. De Schryver, F. C.; De Brackeleire, M.; Toppet, S.; Schoor, M. V. Tetrahedron Lett. 1973, 1253.

5. Kaupp, S.; Angew. Chem. 1972, 84, 259.

6. Leenders, L.; De Schryver, F. C. Angew. Chem. 1971, 83, 359.

7. Leenders, L.; Schouteden, E.; De Schryver, F. C. J. Org. Chem. 1973, 38957.

8. Meerback, M. V.; Toppet, S.; De Schryver, F. C. Tetrahedron Lett. 1972, 2247.

9. Kwanhgee, K. P.; In, K. H.; Joon, W. P. J. Org. Chem. 2001, 66, 6800. 
10. Gupta, S. C.; Yusuf, M.; Arora, S.; Sharma, S.; Kamboj, R. C.; Dhawan, S. N. Tetrahedron 2002, 58, 3095.

11. Gupta, S. C.; Yusuf, M.; Arora, S.; Kamboj, R. C. Tetrahedron 2003, 59, 3609.

12. Gupta, S. C.; Yusuf, M.; Thakur, M.; Kamboj, R. C. J. Chem. Res. (s) 2005, 741.

13. Gupta, S. C.; Sharma, S.; Saini, A.; Dhawan, S. N. J.Chem. Soc., Perkin Trans. 1 1999, 2391.

14. (a) Gupta, S. C.; Yadav, N. S.; Dhawan, S. N. J. Ind. Chem. Soc. 1990, 67, 770. (b) Chem. Abstr. 1991, 115, 159000q.

15. Gupta, S. C.; Saini, A.; Kumar, D.; Yadav, N. S.; Chand, K.; Mor, S.; Dhawan, S. N. J. Chem. Soc., Perkin Trans.1 1995, 177.

16. (a) Wooten, J. B.; Chavdaria, C. G.; Seeman, J. I. J.Org. Chem. 1983, 48, 492. (b) Karplus, K.; Grant, D. M. Proc. Nat. Acad. Sci., USA 1959, 45, 1269. (c) Pachler, K. G. R.; Underwood, W. G. E. Tetrahedron 1967, 23, 1817. (d) Banks, S. W.; Steele, M. J.; Ward, D.; Dewick, P. M. J. Chem. Soc., Chem. Commun. 1982, 156.

17. (a) Hanifin, J. W.; Cohen, E. Tetrahedron Lett. 1966, 5421. (b) Hanifin, J. W.; Cohen, E. J. Am. Chem. Soc. 1969, 91, 4494. (c) Nath, A.; Mal, J.; Venkateswaran, R. V. J. Org. Chem. 1996, 61, 4391. (d) Nath, A.; Venkateswaran, R. V. J. Chem. Soc., Chem. Commun. 1993, 281. 